\title{
AQUAZONE: A Spatial Decision Support System for Aquatic Zone Management
}

\author{
Sekhri A. Arezki \\ Department of Computer Science, University of Oran, BP. 1524 El Mnaouer, Oran, Algeria \\ Email: sekhri.arezki@yahoo.fr \\ Hamdadou B. Djamila, Beldjilali C. Bouziane \\ Department of Computer Science, University of Oran, BP. 1524 El Mnaouer, Oran, Algeria \\ Email: \{dzhamdadoud, bouzianebeldjilali\}@yahoo.fr
}

\begin{abstract}
During the last years, the Sebkha Lake of Oran (Algeria) has been the subject of many studies for its protection and recovery. Many environmental and wetlands experts are a hope on the integration of this rich and fragile space, ecologically, as a pilot project in "management of water tides". Support the large of Sebkha (Lake) of Oran is a major concern for governments looking to make this a protected natural area and viable place. It was a question of putting in place a management policy to respond to the requirements of economic, agricultural and urban development and the preservation of this natural site through management of its water and the preservation of its quality.

The objective of this study is to design and develop a Spatial Decision Support System, namely AQUAZONE, able to assist decision makers in various natural resource management projects. The proposed system integrates remote sensing image processing methods, from display operations, to analysis results, in order to extract useful knowledge to best natural resource management, and in particular define the extension of Sebkha Lake of Oran (Algeria).

Two methods were applied to classify LANDSAT 5 TM images of Oran (Algeria): Fuzzy C-Means (FCM) applied on multi spectral images, and the other that comes with the manual which is the Ordered Queue-based Watershed (OQW). The FCM will serve as initialization phase, to automatically discover the different classes (urban, forest, water, etc..) from a LANDSAT 5 TM images, and also minimize ambiguity in grayscale and establish Land cover map of this region.
\end{abstract}

Index Terms - Fuzzy C-Means, Multi Spectral Image, SDSS, Watershed.

\section{INTRODUCTION}

Oran is the second capital of Algeria and the largest in the Maghreb. It is a port city, located on the southern shore of the Mediterranean, on the north-western Algeria, $432 \mathrm{~km}$ west of the capital Algiers. She stood at the bottom of an open bay on the north of the Gulf of Oran [1]. To the south it is bordered by a large Sebkha (Arabic word, found in desert country is almost a temporarily depression occupied by a lake, usually salted and often where deposited evaporates. The waters come from runoff, but also groundwater according to the dictionary geology [2]).

The great Sebkha in south of Oran, in the watershed of Chott Chergui, is powered by a complex river system from the Murdjajo north and south Tessala. The plains have a great agricultural potential, while the North and South of the Sebkha experienced two different types of development. This river system is the subject of negotiations between supporters of development of rich agricultural plains surrounding the one hand, and defenders of the ecosystem on the other. The northern part of the Sabkha benefited from the expansion and development of the city of Oran and its industrial activity.

It is now an important source of pollution which increases the salinity of the Sabkha. The southern part is rather poorly exploited, there is little infrastructure.

Sectors of water and forest are considered as strategic areas, given their socio-economic impact and their relation with the future of humanity. In last years the town of Oran has known a vast urban extension, and many other land cover changes. In another way the coming years are essentially characterized by global warming which generates a disturbing destabilization of this region with a Mediterranean semi-arid hot variant climate.

This global phenomenon can lead to serious consequences including the drying up of water sources, the change of flowering dates, salinization of soils and deforestation. The Sebkha Lake of Oran is threatened, tree, water and the environment: a matter of challenge.

It therefore seems useful and even urgent to make an inventory to better perceive the future. As well as this study aims to propose an approach to follow-up the Sebkha Lake extension, and the influence of the fast urban evolution and other factors on the ecosystem of this aquatic zone.

Knowledge of our environment and socio-economic data that describe, brought to a location becomes an increasingly critical component of the decision-making regarding the development of our modern society. Thanks to remote sensing, land surface is now scrutinized with an adjustable back that integrates distances and with prodigious capacity to spend the whole retail and juggle time scales and distances. The human had some problems to interpret the images it receives precisely, in the rough, satellite images or other, do not reflect the reality on the ground because the measurements are noisy and low contrast, and difficult to use in geometry, this is due to 
several factors related to the position of the object, the effect of terrain, the transitions between region. The evolution of technology in particular decision support overcomes this problem especially when it comes to the earth observation for studies of climatology, geology or forestry or just land use or natural resource management [3]. Indeed, the interests of rational management of water resources are well established as the agricultural and socio-economic and ecological.

But the decision makers face a complex spatial problems often have multiple conflicting objectives for its solution.

Decision makers are turning more and more towards the Spatial Decision Support Systems (SDSS) to enhance their decision making capabilities and solve complex spatial problems. Conventional systems do not adequately take into account the decision, because they lack the capacity of analytical modeling, and do not easily accommodate the different variations of the process of decision making space.

The purpose of the current study is to create a base for trade-off to the development of several spatial technologies, and solve a large variety of spatial problems. This paper presents a Spatial Decision Support System namely AQUAZONE incorporating remote sensing image processing methods. The designed system, the types of problem to which AQUAZONE can be applied; the decision-making process they support and a framework for the implementation of spatial technologies, and subsequent evolution are examined in this paper. The secondary objective is the development of new automated namely Fuzzy C-Means (FCM) methods, and the Ordered Queue-based Watershed (OQW) algorithm, then test them through a classification of Thematic Mapper (TM) Landsat 5 satellite images of Oran (Algeria).

After presenting some elements of reflection to introduce the context of our study, a state of art highlighting the problems associated with Spatial Decision Support Systems and Multi Spectral Image Classification is proposed in Section 2. This Section introduces the fundamentals of satellite image processing while focusing on some concepts of mathematical morphology which seems useful to recall in this paper. This section also presents a state of the art on the main methods for spatial data mining by giving some definitions, and presenting their advantages and disadvantages and a comparative study. The main works in the context of the Spatial Decision Support related to our contribution are listed in Section 3. Section 4 describes, in its whole, our contribution and presents in detail the functional architecture of the proposed system AQUAZONE. The case study and experimental results are shown in section 5. The last section, summarizes results and sketch directions of future research.

\section{StATE OF ART}

The Sebkha arouses the interest of public authorities, which have mobilized significant resources to its development. Based on these objectives, many projects have been proposed. It is, among other things, the creation of a regional land use plan, the creation of an industrial zone in Tafraoui, a water purification station in El Kerma, an irrigation network in the plain of M'leta south of Oran and the realization of small dams in several nearby towns of Sebkha.

This lake, which has always been of crucial importance, has been the subject of several studies and projects for the consolidation and development. But many initiatives since the colonial period remained without day.

In this context, the Sebkha constitutes a balanced ecological system that requires a developed planning. This water zone that covers an area of 298 square $\mathrm{km}$, is considered as the largest salt lake in the western region of Algeria. Currently, it stores an annual average of about 140 million cubic meters of rainwater. In the fight against pollution, which threatens the wetland, Oran has made the realization of a wastewater treatment plant in El Kerma [4].

A major operation to upgrade and development over several years, will be implemented as part of an overall vision rehabilitation of the Sabkha.

In this work a particular intention is given to the multidisciplinary study (sedimentology, tectonics, hydrogeology, geomorphology, chemistry and biology) made by [5] on the Oran region, which allowed the determination of the conditions of creation and how the Sebkha operates, from Miocene to Recent. The authors cite two important factors that governed the construction of this tectonic depression and climate that have conditioned the drainage system and up later vegetation of the Oran area. The Sedimentological analyzes revealed that the originally lacustrine environment has evolved gradually the Sebkha, reflecting the transition from a subhumid to semi- arid climate.

The authors give a good state of the art, with a history of major geology studies of the region of Oran, and a good comparative study of Sebkhas in the world.

The researchers show that this wetland is fed by water runoff from a large catchment area of about 161000 ha. The flow of rainwater comes for the most part of massive Tessala and Murdjadjo.

The latter poured into the area by many wadis like: Misserghine, El Dalia, Tamselmat, Moguenne, Sidi Mediouni. The more important network of wadis that comes from the Mountains Tessala is Tafraoui, Tamrzoura, haimeur, El ghassoul Besbes.

For a comparative study, and strengthen land data, the same work offers topographic, geological and geomorphologic maps. All structural elements watershed on these cards will serve us to detect fractures in the watershed. Otherwise the same directions can be found at the Sebkha and clearly visible on satellite images watershed line.

The lack of information and studies on groundwater and surface of this city have prompted us to study this theme. In addition, a structural study based on aerial photos and satellite images, enables us to understand the mobility and dynamics of Sabkha. Another problem has deserved our attention, the correlation between the two 
margins (north and south) of the Sabkha: is there a continuity of layers or in the Sabkha.

Whether in an economic context, or in a purely scientific framework (reconstruction of the history of the watershed to understand its mobility and dynamic), Western Algeria has been the subject of several scientific studies since 1830 until today. Only, there are no reported cases on the use of DSS for water management in Algeria. Through the collection and organization of multi-source, (Geographic Information Systems (GIS), and data management approaches, the process described in this paper can, however, be considered as the first step towards the development of SDSS.

This paper presents a multi disciplinary approach that requires a broad review of the spatial technologies (Geographic Information System, Spatial Data mining and Satellite Image Processing) for SDSS design, and identifies challenges and future research directions. The proposed Spatial Decision Support System AQUAZONE is designed to support a decision research process for complex spatial problems, enables the decision makers to negotiate and investigate the possible trade-offs between conflicting objectives and to identify unanticipated, and potentially undesirable, characteristics of solutions.

A variety of methods in one model dedicated to the spatial decision-making, this requires many investigation tools namely:

\section{A. Remote Sensing and Spatial Decision Support}

With the conquest of space and orbiting satellites, the man is able to manage easily the planet. Diversity of observation by satellite imagery allows opportunities to address new problems given the extension of the range of levels of perception now accessible. In fact, the need for accurate and realistic information is necessary to help decision makers in their problems [6].

A well designed Spatial Decision Support System is interactive software that helps decision makers to identify useful information from spatial data information, personal knowledge and business models and solve problems and make decisions [7].

Recently, the web adds another dimension to the SDSS, and a new category of SDSS named Web Spatial Decision Support System (WebSDDS) are developed, the authors in [8] give a good state of the art of emergence of SDSS, their architectures and applications, and discuss the challenges for the future development and deployment of SDSS.

\section{B. Satellite Image Processing and Spatial Data Mining}

In the context of this study, image analysis comes after the steps of acquiring and digitizing, it means a discipline of applied mathematics that studies the images and their transformations, in order to improve their quality or to extract useful information, for moving towards an interpretation of the processed images.

This phase is the more analytical, using, in particular artificial intelligence and Spatial Data Mining techniques (SDM), which offers new opportunities for many Decisional applications [10].
Several processing can be applied on such an image to get the desired information, such segmentation can cut image in these components (region extraction or edge detection) or classification to determine its various classes. Classification and segmentation have nearly same objectives, as the former is another form of component labeling that can result in segmentation of various features in a scene [9].

Analyze an image is to extract characteristic information about objects of interest. It mean, for example, to study the number, distribution, shape or size classes visible from a satellite images. A wide variety of techniques known as segmentation, provides a representation of the image with a limited number of classes, it is generally based on research of local discontinuities, with the detection of regions having characteristics of homogeneity. So the goal of segmentation is to divide the original image into several distinct regions.

The Spatial Data Mining (SDM) is a research area in its own right. In fact, its specificity with respect to traditional data mining is that it takes into account the spatial relationships between objects [11].

Image Classification includes a broad range of decision-theoretic approaches to the identification of images. All classification algorithms including supervised and unsupervised classification are based on the assumption that the image in question depicts one or more features (e.g., spectral regions in the case of remote sensing), and that each of these features belongs to one of several distinct and exclusive classes. The classes may be specified a priori by an analyst (as in supervised classification) or automatically clustered (i.e. as in unsupervised classification) into sets of prototype classes, where the analyst merely specifies the number of desired categories.

Several supervised and unsupervised methods based on mathematics were used for classification: Support Vector Machine [12], K-means clustering [13], Bayesian Classifier [14], Maximum Likelihood [15]. The bioinspired approaches have been also widely used for example different types of Neural Networks as Multilayer Perceptron [16], Genetic Algorithms [17] and Artificial Immune Systems [18] are another example of algorithms inspired from biology.

In recent years, the Chinese made a lot of experimental research work in the field of remote sensing image classification, and try to propose some new classification methods which achieved good results in practice. These new methods are well summarized in [19].

In many vision problems, it is extremely difficult to develop a segmentation algorithm that works correctly in all cases, as well as the human visual system.

In aerial or magnetic resonance brain or satellite image classification, the literature generally involves the efficiency of unsupervised classification algorithm called Fuzzy C-Means (FCM) developed by JC Bezdeck [20].

The Fuzzy C-Means algorithm is an unsupervised classification algorithm. It introduces the concept of fuzzy set in its definition. Each point in the data set is 
affected to each class with a degree of membership classes, and they are, all characterized by their centers of gravity. It is based on the optimization of a quadratic criterion, least squares type. The algorithm requires knowing the number of classes in advance, and it generates the classes through an iterative process by minimizing an objective function [21].

\section{C.Mathematical Morphology}

This section summarizes the techniques of Mathematical Morphology (MM) used in the extraction of knowledge through satellite imagery process. MM is a nonlinear image processing technique born in the 60 s by [22]. Much of this theory was developed at the Centre for Mathematical Morphology (CMM) of the "Ecole des Mines de Paris" [23]. Since then, MM has gained considerable importance. It aims to study the objects according to their shape, size, relationships with their neighbors (especially topological), their texture, and their grayscale or color. MM is at different levels of the image processing process (filtering, segmentation, measurements, texture analysis and provides tools for pattern recognition. MM now has a broad applications in many areas of image processing, both $2 \mathrm{D}$ and 3D, biology and quantitative cytology, medical imaging, aerial and satellite, robotics and computer vision, nondestructive industrial testing (NDT) in studies documents and works of art [24,25].

\section{Watershed Transform}

The watershed line is the basic tool for segmenting images in Mathematical Morphology. Watershed transformation, is due to S.Beucher [26].

Several shortest paths algorithms for the watershed transform with respect to topographical distance can be found in the literature, During the 80s, the authors of [27], S.Beucher and F.Meyer have improved this technique by developing the concepts of markers. The main ones are based on Ordered Queues, repeated raster scanning, a modified union-find algorithm, or a combination of these.

Currently, using Ordering Queue based Watershed algorithm (OQW) appears to be the best solution to quickly build an image of the watershed. Indeed, this algorithm found by F.Meyer [28], is based on a flooding process, it is much more efficient than the classical algorithm using morphological operators.

The watershed has many advantages that explain its success. It is easy to understand transformation, working directly on the image, it don't need the spectral domain or complex representations. The Watershed is also a nonparametric transformation. There is no need to set the values of many parameters to achieve it. This transformation also works well on grayscale images and color or multispectral images, and lends itself well to hierarchical segmentation techniques.

\section{RELATED WORKS}

This paper presents a multi disciplinary work, many studies in different areas are considered.
There are no major cases reported on the use of DSS for water management in Algeria, the only project is the RAMSAR Convention, through bilateral cooperation between Algeria and foreigners. These projects are for the most part, the definition of a Regional Water Plan (RWP) on the subject of Sabkha Oran [29].

The discipline of Land Planning is brought today to balance a growing number of often conflicting goals, defended by a variety of actors (individuals or organizations). Literature associated with the spatial and territorial decision offers little systems that consider this aspect. In this context two main studies are done namely [30,31], the authors have already addressed application of the Multicriteria Spatial Group Decision Support System in Land Planning. In [32], the authors made a good state of the art and proposed an incident information management framework based on data integration, data mining, and multi-criteria decision making. All of these systems incorporate various levels of the multi-criteria analysis tools (MCDA) coupled with GIS, however they consider the criteria to be independent and are unable to model any interaction between them (interchangeability, correlation, preferred length, etc). Recently, the Web has added a new dimension to SDSS and a new category of Decision Support System called Web-based SDSS (WebSDSS) are developed [33], the authors provides a good overview of the emergence of SDSS, its architecture and applications, and discusses some of the enabling technologies and research challenges for future SDSS development and deployment.

For comparative studies, some works done on the classification of Satellite Images of Oran (Algeria) are taken into account; in [34] a new method of mapping the region of Oran (Algeria) using multispectral images is proposed. In [35], researchers used the Kohonen's network classification approach for land cover mapping.

On the other hand many authors have, moreover, examined the use of the Watershed for the management of natural resources of Algeria, trying to define algorithms to generate the watershed from topographic data. In [36], the authors use a GIS to evaluate the physical characteristics of a watershed and their influence on the flow of water (Watershed of Oued El Maleh, Northwest Algeria). In [37], the authors demonstrate the usefulness and impact of the use of LANDSAT TM data for the monitoring of water status of vegetation cover in Algeria.

\section{CONTRIBUTION}

AQUAZONE is a Spatial Decision Support System that can incorporate a diversity of data, opinion, preference and objective. The proposed System allows users to express individual or corporate values and preferences; highlights the degree of imprecision associated with each input and/or technology: Satellite Image Processing, Spatial Data mining and GIS; facilitates structuring of the spatial decision process; reduces several levels of complex information into a single chart; allows examination of trade-off between 
alternatives; and forces examination of inter-relationships between interest.

In secondary objectives, AQUAZONE was designed to ensure the following characteristics and capabilities:

- Provide mechanisms for the entry of spatial data.

- Allow the representation of spatial relations and structures.

- Ability to support large number of spatial analysis techniques.

- Provide performance in a variety of spatial forms such as maps.

- Explicit design to solve complex spatial problem.

- Flexibility, to combine analytical models with data.

- Capacity to explore the solution space by alternatives; an opportunity to support a variety of spatial decision makers.

This section is dedicated to our contribution. We present in detail our approach based on the methods adopted: the FCM and the Watershed.

\section{A. AQUAZONE Description}

AQUAZONE is an interactive system, designed to support decision makers in solving a spatial decision problem and natural resource management. The proposed system allows data visualization, satellite image processing and analysis. Indeed, the main objective is to extract automatically aquatic zones from LANDSAT 5 TM for the region of Oran (Algeria) by fusion methods.

To this end, we will need three channels of Landsat 5 TM insofar as the Fuzzy C-Means are applied to multispectral images to extract regions (forest, urban, sea ...), and then we transform the resulting image in grayscale so that it is treated with the mathematical morphology, which offers several transformations at different levels of image processing (filtering, segmentation, measurement, texture analysis) and defines several tools for pattern recognition namely the Watershed. Once the basin segmented, it is easy to count the number of pixels that belong to it and thus deduce its volume.

To be acceptable, we must combine several investigative tools in a single system. And to do so, we gave a special intention to the SDSS development strategy adapted by [38], and we join the authors, when they demonstrate how to assist decision makers with complex spatial problems, and show how that geoprocessing system must support a decision research process, rather than a more narrowly defined decision making process.

The authors propose architecture for generating good SDSS, adding new capabilities and features in AQUAZONE architecture, facilitated by a modular design.

The architecture of AQUAZONE system consists of a group of three software modules. Each module provides a group of features relating to capacity and there is a module for database and management system, a display and a user interface (see Figure 1).

The AQUAZONE System is a mature way for data visualization, Satellite image processing, spatial analysis, and modeling. The Power of the AQUAZONE lies in its multidisciplinary approach. It does more than just display the data; it contain subsystems to enable the user to be dynamically situated; and enable to analyze and update the information linked to those locations spatially and can further strengthen the other subsystems.

AQUAZONE is a flexible, robust and effective methodology which can be used in different spatial contexts. Its architecture involves an integrated framework of geographical information system (GIS) and a relational Database Management System (DBMS), equipped with interactive communication capabilities between peripheral software tools.

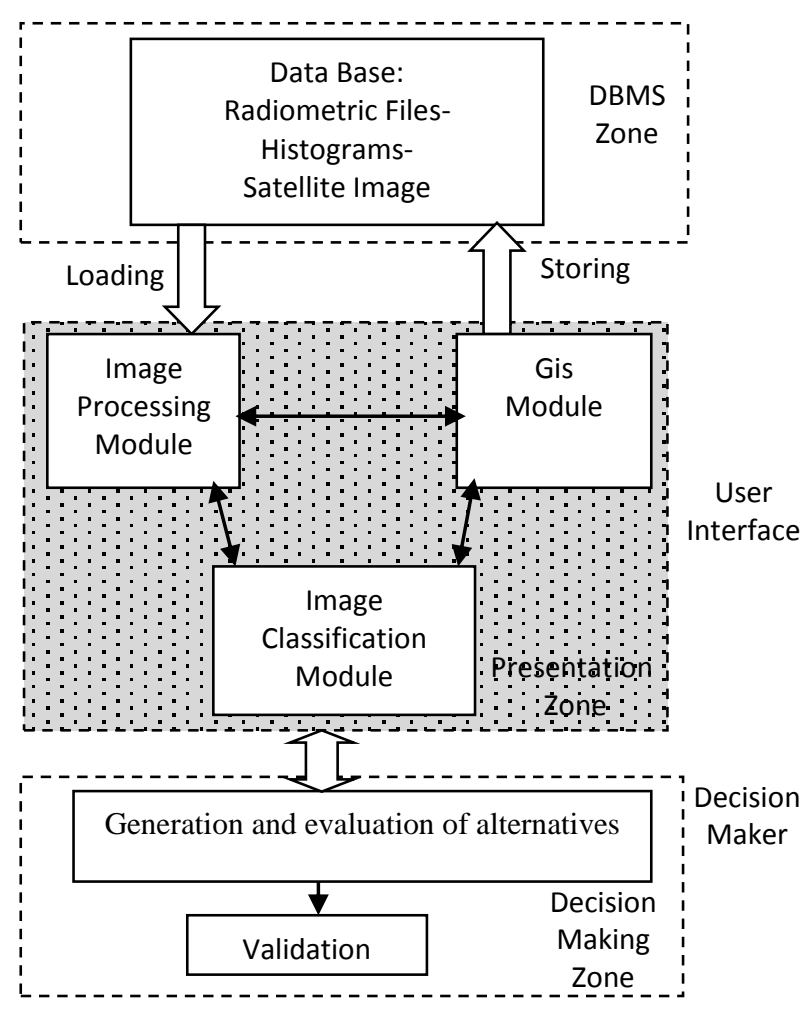

Fig. 1. AQUAZONE: Architecture.

AQUAZONE is structured around three basic components:

\section{a. Database Management System (DBMS):}

The natural resource management requires the integration of a large mass of information from different sources: terrain, satellites, aerial images, maps ... etc.

The DBMS serves as a database for the system, it saves a large amount of data that is the problem class. It must be able to:

- Manage users and stored images, radiometric values, histograms and all results.

- Store and manipulate localization data, topological and thematic, to support map display, spatial query and analytical modeling.

- To inform the user of the data types available and how to access them.

b. Model Based Management System (MBMS):

The MBMS functions are similar to DBMS: 
- Instead of backup data, MBMS backup elements of the models.

- Use an organized structure with a minimum of redundancy and support the representation and exploitation of relationships between elements.

- The algorithmic methods are often used for analytical models. Algorithms constituting these methods consist of a series of steps. When an algorithm is decomposed in part, these steps are the atomic elements (smaller fragment of the algorithm). There remains a common level in the steps used in the algorithms to solve several types of problems of space and non-space analytical models. Combining groups of steps in different sequences, the MBMS is capable of solving a wide range of algorithms.

- The operation of a MBMS requires combinatorial rules algorithm. If steps are atomic, then combinatorial rules may be taken as the formula.

\section{c. User Interface Management System (UIMS):}

AQUAZONE users are often experts in GIS and spatial decision-makers, who have not necessarily mastered the computer tool, the AQUAZONE system must be equipped with an intuitive interface and easy to use. These interfaces help in the creation of models and the interaction between them. The primary function of a UIMS is to improve the use of Spatial Decision Support System, The UIMS of AQUAZONE is composed himself three basic modules:

- Satellite Image Processing Module: contain several image processing techniques (loading images or radiometric values, coloration and region selection, filtering and improving methods as histograms or contrast parameter are needed to noisy or lowcontrast images, contour detection...

- Spatial Data Mining (SDM) Module (Image Classification Module): designed to enable user to integer SDM methods (Supervised/Unsupervised) to extract knowledge from multi-spectral images.

- GIS Module: The GIS subsystem is used as a tool for analysis and decision to interpret the results respecting mapping norms in the form of colored cards with thematic.

The notion of information in AQUAZONE, have the peculiarity of having a spatial dimension, it may be a dimension (rivers), in two dimensions (air and water), or three dimensions (quality of earth, water and air). The nature of this information therefore requires powerful tools offering both opportunities and Analysis. The development of computers and new technologies have enabled to take into account the specificity of these data require different techniques than those used in traditional databases. This leads to develop special software known as Geographic Information System (GIS).

\section{B. The Decisional Model}

The Decision Makers faced to a complex spatial problem often have multiple conflicting objectives for its solution. To be acceptable, a solution must reconcile these conflicting objectives.
This paper aims at finding a compromise between the different methods of spatial analysis to help decision makers to solve problems with multiple criteria. The basic idea is to design a SDSS, combining the methods of classification of images, to deal with different areas (uniform and textured) in image segmentation. This paper introduces a new framework for better classification of Landsat 5 TM multispectral images of the region of Oran (Algeria), based on the combination of methods for automatic extraction of regions (Aquatic, Urban, forest, etc.). The proposed model provides a rational choice between alternatives (GIS, DMS, Multi Spectral image processing).

In this work, the desire to improve the spatial decision making, provided motivation for the development of a powerful decision model, integrating a variety of methods. The originality of our approach is that we consider the superiority of the decision model on human judgment suggests that the best way to improve decision making is to decompose the problem into smaller parts that are simpler and better understood.

The decisional model adopted by AQUAZONE is based on the Decision Support model proposed by [39] and adapted to environmental and Spatial Decision by [40]. The model operates mainly in three phases, namely: The structure of the model, its operations and the achievement of results. Figure 2 illustrates, in detail, the proposed decisional model.

a. Structuring Phase of the Model:

It aims to identify the problem and basic choices on how to approach it. This step is a very sensitive approach throughout the decision support, especially when the method of analysis proceeds by fusion methods, and where treatment is unsupervised. The purpose of this phase is to limit the possibilities, reduce treatment interval, given that the processing occurs on complex data (Multi-Spectral Images).

Structuring is to prepare images, identify actors namely human study that supports the structure and operation of the system, and the decision maker can express his preferences. The main tasks performed during this stage are:

- Formulation of the problem: set initial goals.

- Selection of areas: using LANDSAT TM multispectral image Oran Algeria, which contains, in turn, several regions.

- Load channel 1.3, and 5: each channel contains radiometric values.

- Coloring and Improvement: assignment of colors ( $\mathrm{R}$ (red), G (green), B (blue) to channels $(1,3,5)$ (one for each color channel), and image enhancement...

\section{b. Operational Phase of the Model:}

This is the most analytical decision making step. This is where the methods of Spatial Data Mining will be integrated. In our case, it is essentially the FCM and ordered queue-based watershed.

AQUAZONE the system is dedicated to Geographic Information Systems and spatial experts, the objective of the operation of this work is to provide them with a 
simple, robust, flexible, and efficient tool, which aims to exploitation of FCM and Ordered queue-based watershed.

The main tasks performed during this stage are:

- Display of the colored image;

- Setting the FCM method:

- Set the number of classes.

- Define the partition matrix,

- Choose automatic or manual mode,

- Mathematical Morphology and Ordered Queuebased Watershed

- Graphical display to compare the different results obtained and exploiting the different possibilities.

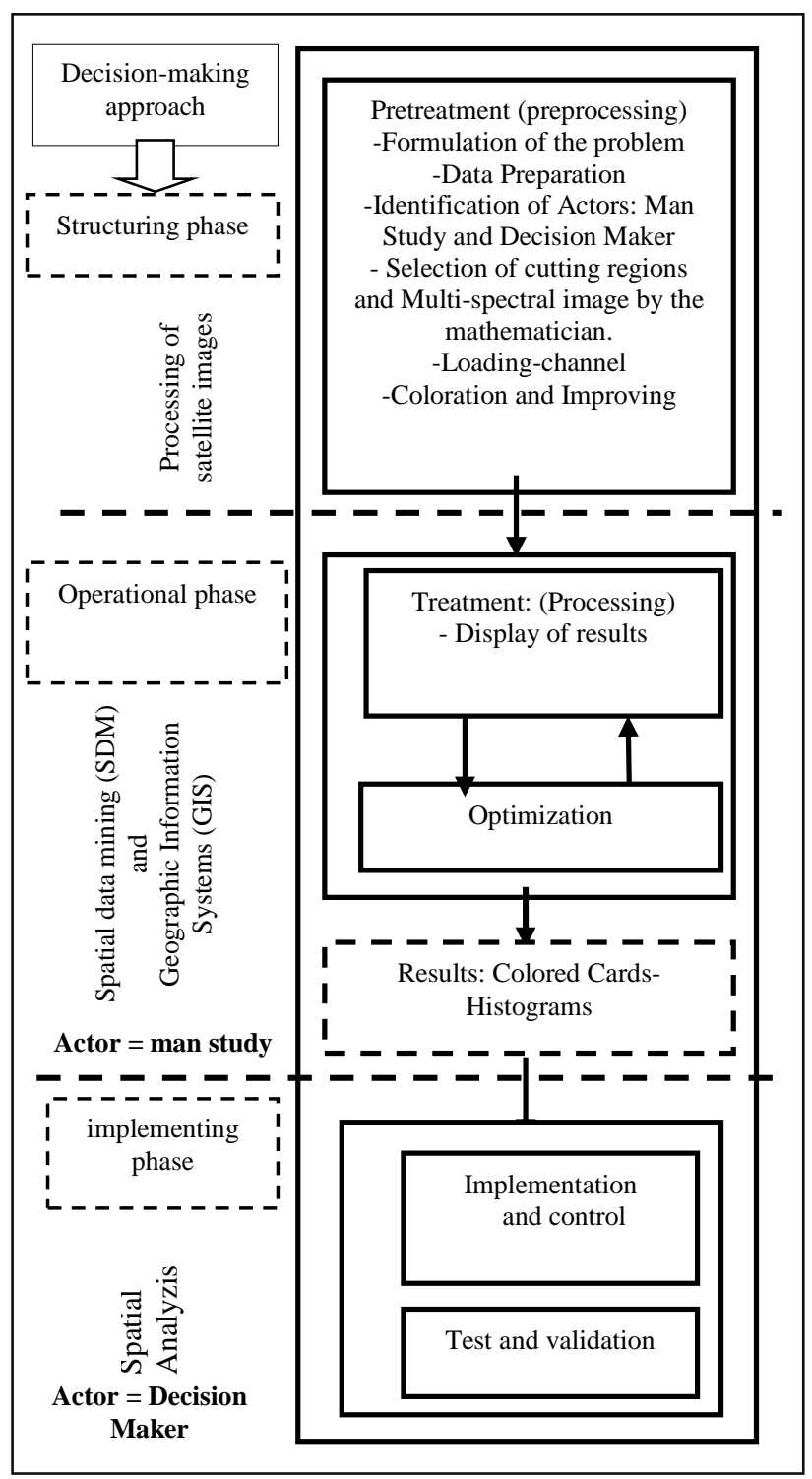

Fig. 2. The proposed decisional model.

\section{c. Implementation Phase of the Model:}

Aims the social acceptance of the result, however, it also includes the implementation of the decision and its control (test and validation).

\section{d. Actors}

The concept of actor refers to a concrete entity, localized (in a context), is a unit of action and decision, made individually or collectively.

In AQUAZONE, we can identify two types of actors:

- The first is the man study, his role is to develop the structure and operation of the model in general is the computer scientist;

- The second actor is the decision maker, he is responsible for the realization of the model, implementation and control of the application, and to test and validate the results.

\section{C.Decisional process}

AQUAZONE was tested on LANDSAT5 TM Multi Spectral image classification; to establish Land Cover map of Oran (West Algeria) and extract Sebkha Lake basin.

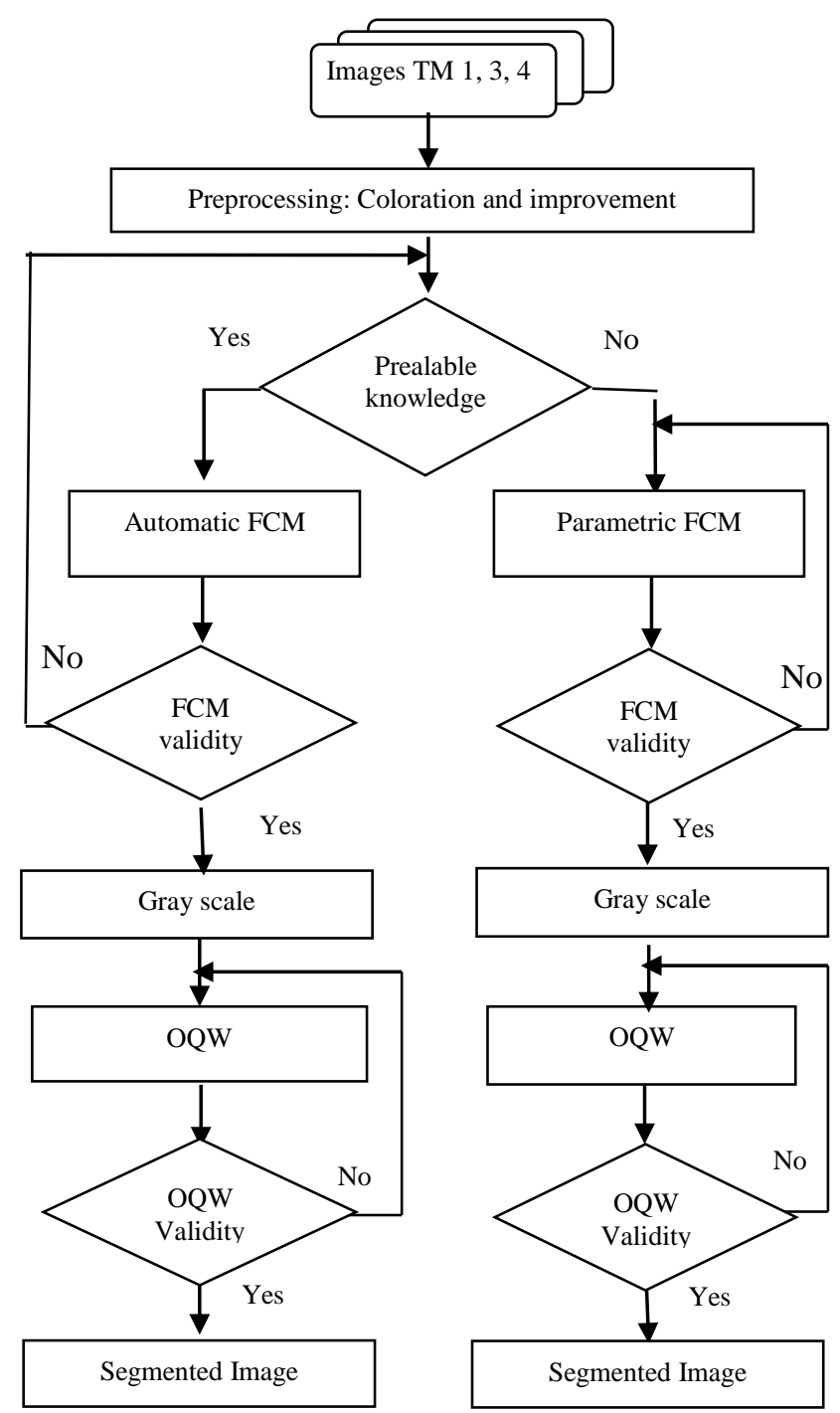

Fig. 3. overall flowcharts FCM-LAND Algorithm

\section{a. General Algorithm of Segmentation}

The segmentation algorithm operates as follows:

Phase 1: Loading images from 3 channels (TM1, TM2,TM3).

Phase 2: Coloring and improvement. 
Phase 3: Image segmentation by FCM method.

Phase 4: Transferring to grayscale.

Phase 5: Segmentation by Ordered Queue-based Watershed Algorithm.

Phase 6: Interpretation and criticism.

A detailed description of the algorithm of segmentation is illustrated by Figure 3.

\section{b. Fuzzy Clustering Methodology}

The Fuzzy C-Means (FCM) algorithm is an unsupervised classification algorithm derived from the concept of fuzzy sets. Every point in the data set for each class with a certain degree of membership and all classes are characterized by their centers of gravity. It is based on the optimization of a quadratic criterion, least squares type. The algorithm requires knowing the number of classes in advance and it generates the classes through an iterative process minimizing an objective function.

The main steps of the FCM algorithm are:

1) Select the number of $K$ classes.

2) Initialize the partition matrix of random centers and using the relation (3)

3 ) Change the partition matrix and the two centers following equations (1) and (2).

We show that minimizing the cost function (5) is performed when the optimality conditions of KuhnTucker conditions are verified [41]:

Let $X$ be a set of vectors $x_{i}(i=1,2 \ldots n)$, where $n$ is the number of pixels in satellite images. And each vector $\mathrm{x}_{\mathrm{i}}$ consists of $d$ feature attributes. In this case, the feature attributes include mean, standard deviation and gray value, i.e. $d=3$. The grouping result according to the fuzzy membership of pixels to classes can be expressed as:

$$
U=\left[u_{i k}\right], 1 \leq i \leq c, 1 \leq k \leq n
$$

Formally, FCM is derived to minimize the following objective function with respect to the membership functions $u_{j k}$ and the centroids $v_{k}$ where $U$ is the fuzzy membership matrix of pixel $k$ in the class i, $c$ is the number of classes. The elements of $U$ satisfy the following constraints:

$$
\begin{aligned}
& u_{i k} \in[0,1] \forall i, k \\
& \sum_{k=1}^{n} u_{i k}=1 \forall k \\
& 0<\sum_{k=1}^{n} u_{i k}<n \forall i
\end{aligned}
$$

The functional of FCM is constructed as follows:

$$
J_{m}(U, V)=\sum_{i} \sum_{k}\left(u_{i k}\right)^{m}\left\|x_{i}-v_{k}\right\|^{2}
$$

Where $\mathrm{v}_{\mathrm{i}}$ is the center of the class $\mathrm{i}, m$ is a real number greater than 1 to control the fuzziness of cluster. The local minimization of functional $J_{f c m}(U, V)$ is done by repeatedly adjusting $u_{i k}$ and $v_{i}$ according to the following relation:

$$
u_{i k}=1 / \sum_{j=1, c}\left(\frac{d_{i k}}{d_{i j}}\right)^{\frac{2}{m-1}}
$$

Where:

$$
\begin{aligned}
& d_{i k}=\left\|x_{i}-v_{k}\right\| \\
& d_{i j}=\left\|x_{i}-v_{j}\right\|
\end{aligned}
$$

This condition is the update of membership degree (9)

$$
v_{k}=\frac{\sum_{i}\left(\left(U_{i k}\right)^{m} x_{i}\right)}{\sum_{i}\left(U_{i k}\right)^{m}}
$$

\section{c. Ordered Queue-based Watershed (OQW)}

In image processing, Watershed is a family of image segmentation methods from Mathematical Morphology (MM) who consider a grayscale image as a topographic relief, it simulate the flood. The classical algorithm is extremely slow, it is based on morphological operators applied on the entire image. The ordered Queue-based Watershed (OQW) is currently the best solution to accelerate the watershed. It performance on standard machines are a serious alternative to dedicated processors that is why this algorithmic structure was chosen.

The principle of calculating the watershed is simple, it usually uses the flooding process, a particular description of the image seen as a topographic relief, where clear structures are peaks terrain and dark structures correspond to valleys relief. We imagine that this topographic surface is perforated at the locations of minima. Then slowly plunge this surface into a lake (supposed infinite expanse of water for the convenience of the experiment). The water will pass through the holes, starting by the deepest minima, and we assume that for each minimum source water hue of a color (minimum label) (see Figure 4).

Each minimum gives rise to a lake increasingly extended as the water level rises and will gradually flood relief [42].

The Ordered Queue is used to quickly create the Watershed of a function f controlled by a set of Markers M, each marker is labeled and each watershed under construction will keep the marker label. Any label can be decomposed into several connected components as each component has a common label [43].

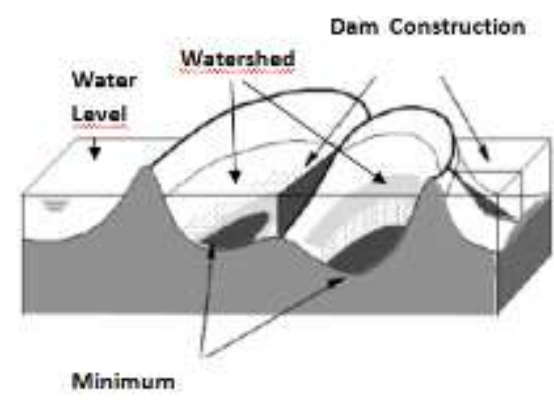

Fig. 4. Principle of flood relief. 
The algorithm of OQW operates as follows:

- The image is interpreted as a topographic relief: Grayscale corresponds to the altitude

- The contours correspond to the crest lines of the relief

- Markers are placed in the picture: they point to the regions to be segmented in the image.

- The relief is flooded, the water entering the terrain through markers flood-sources (it is not about runoff but instead of water welling minimum).

- To prevent the mixing of waters from different minima, we create a basic barrier at each point of contact. Water continues to rise.

- In the end, only stay dams completed surrounded by water: the contours are defined as Watershed line separating the lakes (watersheds). Figure 5 summarize the steps of the OWQ algorithm.

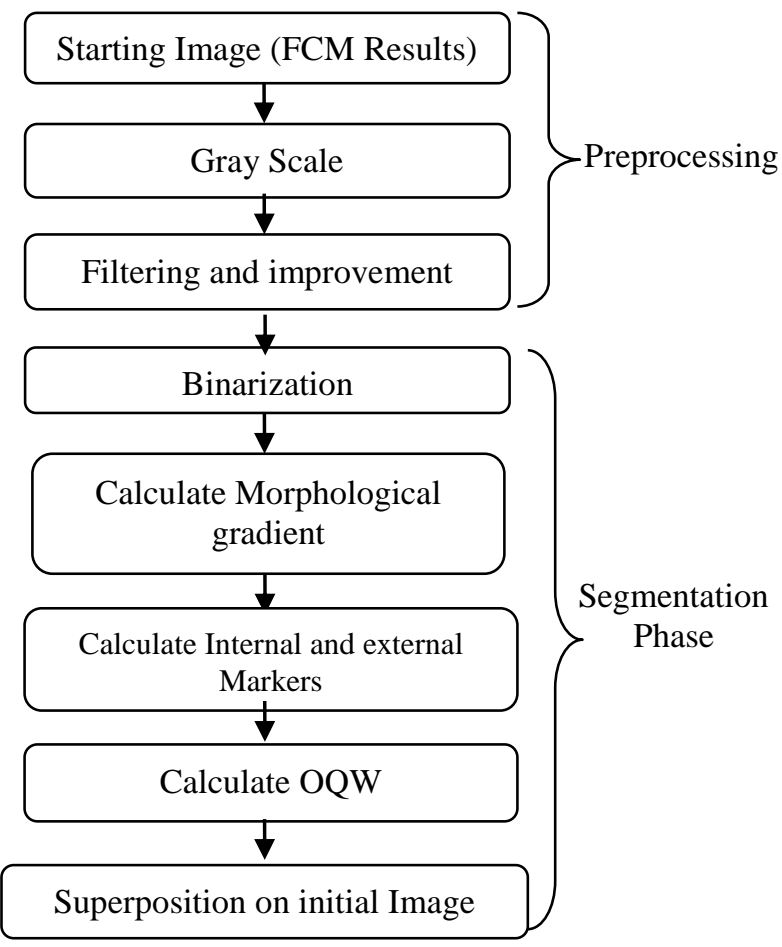

Fig. 5. Steps of the Ordered Queue based Watershed algorithm.

\section{EXPERIMENTAL RESULTS}

The objective of this work is to develop a Spatial Decision Support System AQUAZONE that helps decision makers to automatically extract the various components parts of the region of Oran and establish Land cover map of West Oran (Algeria), to finally separate water areas.

The Great Sebkha is a Salt Lake that is located in Algéria, "wilaya of Oran", "Daira of Boutlelis", and it is distant $12 \mathrm{~km}$ from the Mediterranean Sea and $15 \mathrm{~km}$ from the city of Oran. It has approximately $44 \mathrm{~km}$ by $12 \mathrm{~km}(52800 \mathrm{Ha})$. AQUAZONE aims to set up a classification on satellite images of Oran (West Algeria, size $1024 * 1024$ (Figure 6), acquired by the LANDSAT 5 satellite in March 15, 1993 at 9:45 am. [44].
Experimental results on real images demonstrate that the proposed system is able to improve the compactness of the clusters forming uniform regions and extract useful information for best resource management decision making.

\section{A. Nature of data}

The AQUAZONE was tested for Windows platforms, focusing on the detailed satellite image of Oran Algeria.

The zone of study is chosen for the diversity of themes characterizing it (water, forests, urban areas, cereal, etc...) and its relatively rugged terrain and variable relief.

The objective of AQUAZONE is to segment the original image by FCM, which needs the three TM channels 1, 3 and 4 of LANDSAT TM images. These are files containing radiometric values for each pixel in the image, as FCM are applied on multispectral images.

The used bands are: red, infrared and blue. In our application we used a portion of the study area Sebkha Lake of size $400 * 400$ (area shown in Figure 6).

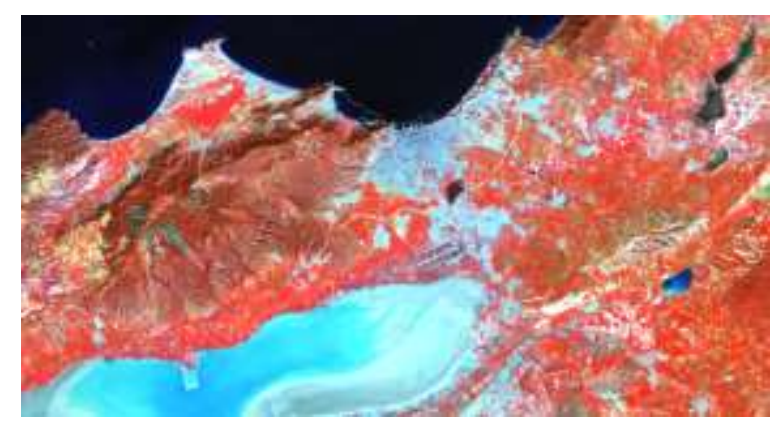

Fig. 6. Zone Study: LANDSAT 5 TM image of West Oran Algeria.

\section{B. AQUAZONE Running}

The proposed system proceeds in several steps:

\section{a. Preprocessing}

This step provides several tasks:

- Formulation of the problem: set initial objectives.

- Downloading channels 1,3, and 4: each channel contains Radiometric values.

- Coloring and Improvement: color assignment ( $R$ (red), G (green ), B ( blue) to the radiometric files $(1,3$,and 4$)$ of the satellite LANSAT 5 ,

- Region choice and enhancement: cutting the multi spectral LANDSAT 5 TM image of Oran Algeria.

- Display the image of Sebkha Lake (See Table 1 Step 1).

\section{b. Treatment:}

The objective of this step is to improve the segmentation in very few areas, then detect the most significant from the gradient contours; contours that are robust to changes in conditions and segmentation that can separate water regions than other components themes Oran region.

This step is synonymous with the exploitation stage of the Decision model, it operates in four steps:

\section{FCM}


FCM gives a very interesting result for our application. In our work, we applied the FCM on multispectral colored images (RGB).

Several tests were done with different settings of FCM (Number of classes, performance matrix), but the best results nearest to real land data, is obtained with the following parameters:

We fix $m=2$, and the number of class $K=10$, and a fair degree of membership Uik $=0.1$ for classes. The results obtained after segmentation are shown in Table 1 Step 2.

\section{Grayscale Transfer}

The grayscale transfer consists to extract information for the three RGB colors from the color image. The figures below show the automatic transfer of the results obtained by the FCM segmentation to grayscale; this step is necessary because the watershed is applied on mono spectral images.

The (Table 1 Step 3) shows the grayscale transfer for the original image.

The (Table 1 Step 4) shows the grayscale transfer of the results obtained by the FCM automatic segmentation,

\section{Watershed Segmentation}

Regions obtained by a first level of FCM segmentation can be processed again by OQW, to collect homologous regions. The OQW is a method based on Mathematical Morphology (MM). It presents a set of algorithms applied to gray level images. The objective is to extract the Most significant minima, according to a morphological criterion (contrast, area, volume), which implies a hierarchy based on the extinction values. The Lake is automatically extracted from the LaANDSAT 5 TM satellite image of Oran (see Table 1, Step 5 to Step 9).

\section{Fusion: FCM-OQW and Superposition}

This step allows the superposition of the line of the watershed on the original image or other results (segmented image by FCM for example). AQUAZONE offers several alternatives of fusion, allowing the decision makers to have more choice, more results, for better analysis and better decision-making (see Table 1, Step 10).

By analyzing different images obtained by the different tests, the AQUAZONE system enable us to quantify the occupied surface for each class (sea, urban, vegetation...etc). The classification rate obtained by FCM result (Table 5.1 Etape 2: FCM segmentation $\mathrm{K}=10$ ), is shown in Table 2 .

Table 2. Classification Rate obtained by FCM $(\mathrm{K}=10)$

\begin{tabular}{|c|c|c|}
\hline $\mathrm{N}^{\circ}$ & Class & Classification Rate \\
\hline 1 & Water & $8.17 \%$ \\
\hline 2 & Urban & $16.22 \%$ \\
\hline 3 & Fallow Land & $7.64 \%$ \\
\hline 4 & Agriculture & $10.63 \%$ \\
\hline 5 & Scrubland & $16.87 \%$ \\
\hline 6 & Sebkha (Lake) & $18.56 \%$ \\
\hline 7 & Sand & $2.56 \%$ \\
\hline 8 & Amber & $2.23 \%$ \\
\hline 9 & Forest & $15.48 \%$ \\
\hline 10 & Bare soil & $1.64 \%$ \\
\hline
\end{tabular}

Table 1.Obtained Results

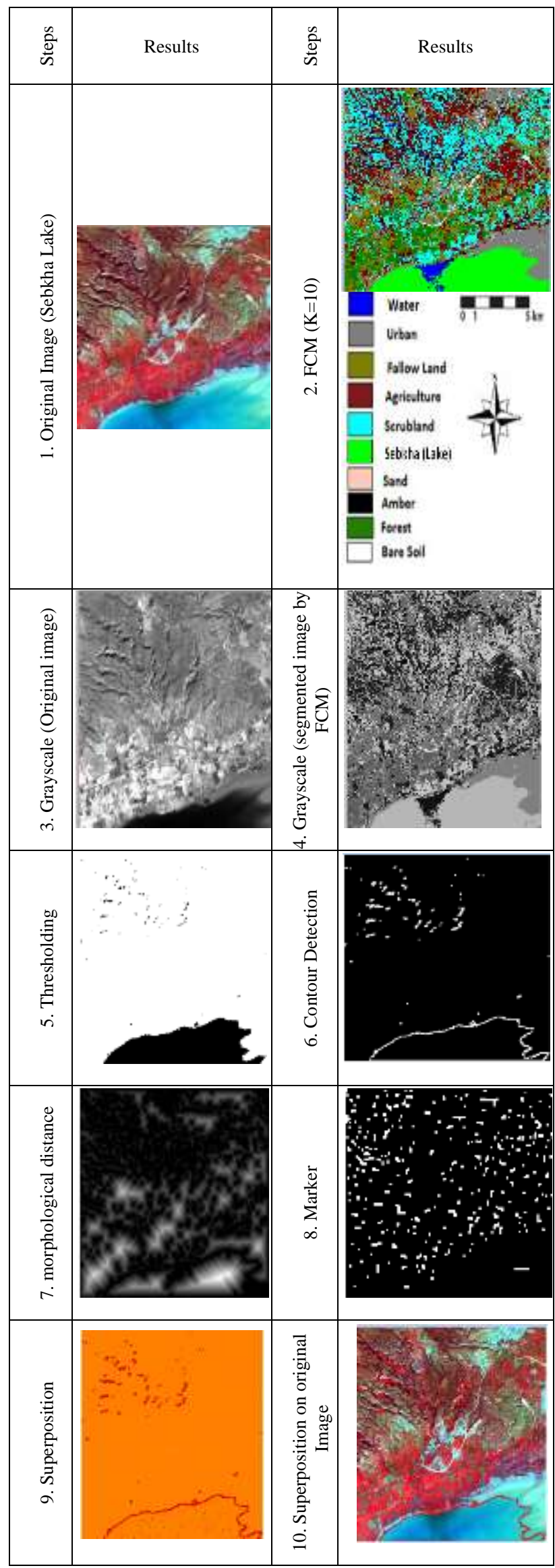




\section{c. Interpretation of Results and Criticism}

The proposed System AQUAZONE is an interactive system, computerized and designed to support a user or group of users in achieving a higher effectiveness of decision making while solving a spatial decision problem. AQUAZONE was tested on real LANDSAT5 Satellite images of Oran Sebkha side. This region is known for the variety of themes present (water, forest, cereal, urban areas, etc...) and its relatively rugged terrain. By comparing the results obtained with the real ground data, that we have little information, and after the evaluation of the classification error rate achieved by different structures for different sets of samples, we found that the proposed approach gives good results.

This work allows, in a first time to extract traces of aquatic areas, and an inventory changes for the Sebkha area, can be made by treating multi-date satellite images. This is beneficial to follow the evolution and changes of the watershed line, which are due to several factors (global warming, or town planning, pollution or other... ).

And from these results we noted that:

- The studied algorithm gives significant good result but it has two drawbacks: first, they require the prior choice of the number of classes $\mathrm{K}$, which makes it impossible to automate the process, and secondly, it often requires a high calculation time, because it's an iterative algorithm.

- Each time we increase the number of classes, the result of FCM is better, but requires high computation time. The problem also persists when we use large images. This problem can be reduced by using a powerful hardware.

- The choice of the membership degree has an important role to partition regions. This choice is the problem of deprivations of regions when we give degrees without prior knowledge in the field. To remedy this problem, we reformulated the relationship of this algorithm by adding expert knowledge to automate processing. We found that with the automation of the algorithm we eliminate all the disadvantages of the FCM.

- From the tests we noted that the FCM is effective when the luminance variation is too large (we are talking about the concept of texture). The result of this investigation shows that the underlying structures and patterns from satellite image data can be classified more precisely than the conventional ones.

- The OQW has a remarkable advantage: most of transformation steps can be easily illustrated, and results are immediately seen, even if the underlying algorithms can be complex. We observe in ( Table 1, Step 10) that the OQW gives rise to a less extended on the east of the lake side, as this area is shallow (near maxima) as the water level rises and will gradually flood relief.

- Remains the problem of complexity of the algorithms namely FCM and OQW and their execution time. We then see the interest to develop new algorithms to reduce the processing time. The
OQW is one, but it is not the only one. There are even here many approaches. It is likely that the solutions depend largely on the nature and size of the processed images.

- This approach also has another advantage: it indicates the direction that should be worn efforts to obtain even better segmentation algorithms.

- From the results we can remark an incertitude in the bottom east zone of the sebkha lake Image classification (see Table 1, Step 2), This interesting region that the bottom of the depression at 80 meters above sea level has a slight asymmetry in the eastern part. After analyzing, we found that the reason is that the Sebkha is fed by the runoff of the watershed, the water of this wetland is salty. This is due to climatic effects where alternating periods of flooding with those draining that characterize the Sebkha. The evaporation of water leads to stockpiling salt, another part is fed by the wind from the depression to the peripheries.

The water that is salty, it forms a film of 10 to $30 \mathrm{~cm}$, which varies depending on rainfall. This film completely dry during the summer following a very high evaporation and a drought that has lasted a decade.

\section{CONCLUSION}

We presented a Spatial Decision Support System AQUAZONE, this system is a combination of geographic information systems (GIS), technical features of remote sensing, mathematical models and other decision support features. This combination allows system users, decision makers, using this tool to model and analyze the data and view the output on thematic maps.

The success of AQUAZONE returns to its architecture quality, both simple and modular, allowing a specialist, to locate and apply these methods directly following the decision approach proposed, it can also negotiate with other experts in other modules for better decision making. The system unites various informations, in order to give them a large size to produce and view thematic maps for a best geographic data management and decision support.

Experiment results show that the approach is highly effective on multi-spectral images, and the underlying structures and patterns from satellite image data can be classified without really having the best of available resources (time, data, experts...).

Whether for Oran or other area, the category of natural resource management, contains a wide range of application domain of AQUAZONE, above all, water management, or exploitation of forests. In the context of water resources, it handles requests estimates of irrigation, watershed management (Mediterranean Sea and Sabkha Lake). Still applying AQUAZONE on disciplines dealing with aquatic biodiversities, management and regulation of coastal and seabed habitat conservation, ecological restoration of coastal areas... so that the SDSS AQUAZONE is more effective, and offers to the experts in territory planning, an efficient tool to follow up the evolution of various spaces. 
A number of possible orientations can be identified when we look at the prospects for the development of AQUAZONE. The improvement in this system by integrating new feature in the general structure, or by testing other segmentation and optimization algorithms should increase the number of people who could easily use it for decision-making. Future developments are likely to include all of these trends, with different groups of users who benefit from these changes.

For perspectives, we propose to test this System on other Multi spectral images, and adapt it to other types of images (Aerial, Hyper Spectral) and make comparative studies.

\section{REFERENCES}

[1] B. Augustin "Oran étude de géographie et d'histoire urbaines”, ,ISBN 9789961953310, p. 414, 1939.

[2] A. Foucault, J.F.Raoult, Dictionnaire de Géologie, Dunod 5è édition, 379p. France, 2001.

[3] V. Mesev, "Integration of GIS and remote sensing", 2008.

[4] Benziane and al,. "Aptitude des eaux du bassin de la Grande Sebkha d'Oran á l'irrigation", Journal of Applied Biosciences 56: 4066-4074, ISSN 1997-5902, 2012.

[5] M. Kacem, "Etude d'une Sebkha : la Sebkha d'Oran (Ouest algérien)", Phd Thesis PhD thesis in Earth Sciences Option: Sedimentology,Oran Algeria, 2006.

[6] J. A. Richards, "Remote sensing digital image analysis: an introduction", ISBN 978-3642300615, 2013.

[7] J. P. Shim, M. Warkentin, "Past, present, and future of decision support technology", Decision Support Systems, Elsevier, 931, 2002

[8] V. Sugumaran, R. Sugumaran, "Web-based Spatial Decision Support Systems (WebSDSS): Evolution, Architecture, and Challenges", Proc. Third Annual SIGDSS, Pre-ICIS Workshop Designing Complex Decision Support: Discovery and Presentation of Information and Knowledge, Las Vegas, Nevada, 2005.

[9] R. G. Congalton, L. K. Fenstermake, I. R. Lensen, "Remote Sensing and Geographic Information System Data Integration: Error Sources and Research Issues", Photogrammetric Engineering \& Remote Sensing, Vol. 57, No. 6, June 1991, pp. 677-687

[10] S. Shekhar, Y. Huang, W. Wu, C.T. Lu, "What's Spatial about Spatial Data Mining: Three Case Studies, as Chapter of Book: Data Mining for Scientific and Engineering Applications”, 2001, ISBN 1-4020-0033-2.

[11] D. J. Hand, "A Practitioner's Guide to Resampling for Data Analysis, Data Mining, and Modeling", International Statistical Review, Vol:81, ISSN:0306-7734, 2013, pp:326-326.

[12] S. Moustakidis, G. Mallinis, N. Koutsias, J. B. Theocharis, V. Petridis, "SVM-Based Fuzzy Decision Trees for Classification of High Spatial Resolution Remote Sensing Images", IEEE Transactions on Geoscience and Remote Sensing,. Vol. 50 , No. 1, 2012 , pp. 149-16.

[13] D. X. Chang, X. D. Zhang, C. W. Zheng, "A genetic algorithm with gene rearrangement for K-means clustering", Pattern Recognition, Vol: 42, No.7, 2009, pp. 1210-1222.

[14] J. Huang, Y. Yuan, "Construction and Application of Bayesian Network Model for Spatial Data Mining", Control and Automation, IEEE International Conference on Digital Object, Id 10.1109/ICCA.2007.4376872 , 2007, pp. $2802-2805$.
[15] Z. Wang, J. R. Jensen, I. Jungho, "An automatic regionbased image segmentation algorithm for remote sensing applications", Environmental Modeling and Software, Vol. 25, No. 10, 2010, pp. 1149-1165.

[16] L. H. Thai, T. S. Hai, N. T. Thuy, "Image Classification using Support Vector Machine and Artificial Neural Network", I.J. Information Technology and Computer Science, 2012, 5, 32-38.

[17] S. Bandyopadhyay, S. K. Pal, "Pixel Classification Using Variable String Genetic Algorithms with Chromosome Differentiation". IEEE transaction on geoscience and remote sensing, vol. 39, n. 2, pp 303-308. February 2001.

[18] B. Gong, J. Im, G. Mountrakis, "An artificial immune network approach to multi-sensor land use/land cover classification", Remote Sensing of Environment, Vol. 115, No. 2, 2011, pp. 600-614.

[19] Z. Hebing, W. Shidong, "Research Progress of Computer Automatic Classification Technology and Methods Based on Remote Sensing Images", Advances in Intelligent Systems Research, 2012, ISBN: 978-94-91216-00-8.

[20] J.C. Bezdek," Fuzzy mathematics in pattern classification", Cornell Univ, 1973.

[21] X. Li, S. Bian,” Multiresolution Fuzzy C-Means Clustering Using Markov Random Field for Image Segmentation", I.J. Information Technology and Computer Science, 2009, 1, 49-57.

[22] J. Serra. "Image Analysis And Mathematical Morphology", Academic Press, 1982.

[23] S. Beucher, “ segmentation d'image et morphologie mathematique ", cours du Master, St Etienne, CMM, Mines Paris Tech, 2010. http://cmm.ensmp.fr/ beucher/ publi/Segmentation_SB_print.pdf.

[24] J. Serra. "Image Analysis And Mathematical Morphology. Theoretical Advances". Academic Press, 1988.

[25] G. Noyel, J. Angulo and D. Jeulin. "A new spatio-spectral morphological segmentation for multispectral remote sensing images". International Journal of Remote Sensing , 2010.

[26] S. Beucher. "Segmentation D'images Et Morphologie Mathématique". Phd thesis ,Centre De Morphologie Mathématique, Ecole Des Mines De Paris, Juin 1990.

[27] S. Beucher, F. Meyer,"The Morphological approach To ImageSegmentation: The Watershed Transformation". Mathematical Morphology In Image Processing, E. Dougherty (ed.), ch. 12, Marcel-Dekker, New York, Pages 433-481, 1993.

[28] F. Meyer, "Un Algorithme Optimal De Ligne De Partage Des Eaux “, 8 ème Congrès De Reconnaissance Des Formes Et Intelligence Artificielle, Pages 847-857, Novembre1991.

[29] Nostrum-DSS Project, NOSTRUM-DSS "Algerian National Report", Contract N.:INCO-CT-2004-509158. www.nostrum-dss.eu. http://ramsar.wetlands.org /Portals /15/ALGERIA.pdf.

[30] D. Hamdadou, T. Libourel, "A MultiCriteria Group Decision Support System for Industrial Diagnosis" INFOCOMP, v.10, No. 3, P. 12-14, September of 2011.

[31] D. Hamdadou, K. Bouamrane, "Towards a Multicriteria Spatial Group Decision Support System, Application: Territory Planning", ICEMCS 2012, IEEE, 2012, Tanger, Maroc.

[32] Y. Peng, Y. Zhang, Y. Tang, S. Li, "An incident information management framework based on data integration, data mining, and multi-criteria decision making".Decision Support Systems, Volume 51, Issue 2, May 2011, Pages 316-327. 
[33] V. Sugumaran, R. Sugumaran, "Web-based Spatial Decision Support Systems (WebSDSS): Evolution, Architecture, and Challenges", Third Annual SIGDSS PreICIS Workshop Designing Complex Decision Support: Discovery and Presentation of Information and Knowledge, Las Vegas, Nevada December 11, 2005.

[34] F. Laoufi, A. H. Belbachir, N. Benabadji, A. Zanoun ,“A new method of mapping the region of Oran (Algeria) using multispectral remote Sensing" C. R. Geoscience, Vol 343, 2011, pp 653-663.

[35] T. Iftene, H. Mahi, H. Isabaten, “The Kohonen's Network Classification Approach Used For Land Cover Mapping: Case of Oran Area (Algeria)", Télédétection, Vol. 3, n 23-4, 2003, pp. 361-372.

[36] N. Bentekhici, “Utilisation d'un SIG pour l'évaluation des caractéristiques physiques d'un bassin versant et leurs influences sur l'écoulement des eaux (bassin versant d'Oued El Maleh, Nord-Ouest d'Algérie“, Conférence Francophone ESRI, Issy les Moulineaux, SIG, 2006.

[37] A. Hamimed, K. Mederbal, A. Khaldi, "Utilisation des données TM de Landsat pour le suivi de l'état hydrique du couvert végétal en Algérie“, Proc. VIIIème Journées Scientifiques de Télédétection, Lausanne, 25-28 Nov. 1999.

[38] P. J., Densham, "Spatial Decision Support Systems", 1991.

[39] J. Pictet, "Dépasser l'évaluation environnementale, procédure d'étude et d'insertion dans la décision globale". Collection Meta, Presses polytechnique et universitaires Romandes, Vol. 1015, Lausanne, suisse, 1990.

[40] D. Hamdadou, "Un Modèle pour la prise de décision en Aménagement du Territoire : Une Approche Multicritère et une Approche de Négociation". Phd Thesis, Oran University, Algeria, 2008.

[41] J. C. Bezdek., "A convergence theorem for the fuzzy ISODATA clustering algorithms", IEEE Trans. Pattern Anal. Machine Intell. 2,1980.

[42] J.Angulo, «Approche probabiliste à la segmentation morphologique par LPE, avec des exemples en imagerie multi-variée », Séminaire au GIPSA-Lab, INPGrenoble, 2007.

[43] P. Soille, "Morphological Image Analysis - Principles and Applications", Springer-Verlag, 1999.

[44] Z.Smahi, K. Benhanifia, A. Bensaid, "Development of an algorithm of absolute atmospheric correction for Multitemporal satellite images. Application to the region of oran (west of algeria)", international archives of photogrammetry and remote sensing. Vol. Xxxiii, part b7. Amsterdam 2000.

\section{Authors' Profiles}

A. Sekhri is Phd Student at the Computer Science Department, University of Oran Algeria, Teacher at Management Science Department, University of Oran 2 Mohamed Benahmed, Algeria. He received his Engineering degree in Computer Science and his Master from the Computer Science Department in 2008 and 2011.His research interests includes Spatial Decision Support Systems, Geographic Information Systems, Satellite Image Processing and Spatial Data Mining.

D. Hamdadou received her Engineering degree in Computer Science and her Master of Science degree from the Computer Science Institute in 1993 and 2000, respectively. She also obtained her doctorate in 2008. She received her $\mathrm{PhD}$ in 2012 from the Computer Science Department of university of Oran. Her research interest includes Decision Support System, Geographic Information System, Multi Criteria Approaches.
B. Beldjilali is a Professor at Computer Science Department University of Oran, he received his Engineering degree in Computer Science from the Polytechnic National School of Algiers in 1973, Director of post-Graduation "Computer Science and Automatic" from 1999 to 2009 and actually is director of Computer Science Laboratory of Oran (LIO). His research includes: Expert Systems, Deductive Data Bases, and Currently the Production Systems (Management and Control) and Knowledge Management.

How to cite this paper: Sekhri A. Arezki, Hamdadou B. Djamila, Beldjilali C. Bouziane,"AQUAZONE: A Spatial Decision Support System for Aquatic Zone Management", International Journal of Information Technology and Computer Science(IJITCS), vol.7, no.4, pp.1-13, 2015. DOI: 10.5815/ijitcs.2015.04.01 